\title{
Latex glove industry: prevalence of heat-related illness among Malaysian workers
}

\begin{abstract}
Glove production involves work processes with a high temperature. However, few studies have been conducted on the hazards of high temperature among workers. This study was conducted to evaluate the prevalence of heat-related illness among glove industry workers under heat stress conditions. This cross-sectional study was conducted in a glove factory at Selangor. The total number of workers $(n=82)$ were interviewed using adapted questionnaires. The highest prevalent heat-related illnesses in respondents were dehydration $(82.2 \%)$, followed by heat exhaustion $(81.1 \%)$, heat cramps $(23.2 \%)$, heat rashes $(22.2 \%)$, heat stroke (5.6\%), and heat syncope (4.4\%). The workers are highly exposed to dehydration and heat exhaustion illness. Therefore, prevention of heat stress is very important in this industry. It is the duty of the employers to implement suitable prevention measures to protect the health and safety of their workers. As a recommendation, personal protective equipment such as a cool vest also helps in protection from the hot working environment. The cool vest has inserted frozen liquid placed between the fabrics and is worn on the upper trunk. This helps in giving workers freedom to move around, as it has no attached lines. This suit has a constant and steady cooling effect on the whole body. Besides this, training on heat stress and on the ways of controlling heat stress should be provided by employers. This training program can help in educating and creating awareness among workers on the symptoms of heat-related illness, which can help in preventing any worst case of heat stress from occurring. The training program may include the description of heat stress, including the symptoms of heat-related illness and the first-aid measures for each disorder.
\end{abstract}

Keyword: Dehydration; Glove industry; Heat exhaustion; Heat-related illness; Heat stress 